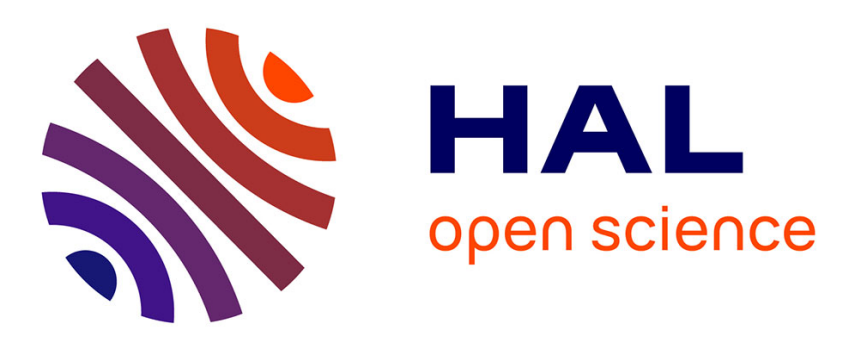

\title{
Integration of extra-information for belief function theory conflict management problem through generic association rules
}

\author{
Ahmed Samet, Eric Lefevre, Sadok Ben Yahia
}

\section{- To cite this version:}

Ahmed Samet, Eric Lefevre, Sadok Ben Yahia. Integration of extra-information for belief function theory conflict management problem through generic association rules. International Journal of Uncertainty, Fuzziness and Knowledge-Based Systems, 2014, 22 (04), pp.531-551. 10.1142/S0218488514500263 . hal-03354150

\author{
HAL Id: hal-03354150 \\ https://hal.science/hal-03354150
}

Submitted on 29 Sep 2021

HAL is a multi-disciplinary open access archive for the deposit and dissemination of scientific research documents, whether they are published or not. The documents may come from teaching and research institutions in France or abroad, or from public or private research centers.
L'archive ouverte pluridisciplinaire HAL, est destinée au dépôt et à la diffusion de documents scientifiques de niveau recherche, publiés ou non, émanant des établissements d'enseignement et de recherche français ou étrangers, des laboratoires publics ou privés. 


\title{
Integration of extra-information for belief function theory conflict management problem through generic association rules
}

\author{
Ahmed Samet \\ Laboratory of research in Programming, Algorithmic and Heuristic \\ Faculty of Science of Tunis, Tunisia \\ ahmed.samet@fst.rnu.tn \\ Eric Lefèvre \\ Univ. Lille Nord de France UArtois, EA 3926 LGI2A \\ F-62400, Béthune, France \\ eric.lefevre@univ-artois.fr \\ Sadok Ben Yahia \\ Laboratory of research in Programming, Algorithmic and Heuristic \\ Faculty of Science of Tunis, Tunisia \\ sadok.benyahia@fst.rnu.tn
}

\begin{abstract}
Decision making by considering multiple information sources could provide interesting results. For that reason, fusion formalisms were a major concern in the belief function community. In this context, the Belief function theory allows information fusion thanks to its combinations tools that it integrates. Nevertheless, belief function theory highlights a limit in the merging of contradictory (conflictual) sources. Many authors tackled this problem offering contributions in this field. Unfortunately, no proposed operator has distinguished by its adequacy regardless the type of handled sources. In this paper, we demonstrate the limits of some referenced works and we diagnostic the issues origin. We propose a conflict management approach based on an extra-information that guides the treatment. We also integrate a generic associative base borrowed from the data mining
\end{abstract} domain in order to apply the adequate conflict management.

Keywords: Belief function theory; Conflict management; Generic base; Associative classification

\section{Introduction}

The belief function theory provide an adequate formalism for uncertainty modeling. ${ }^{1,2}$ Worth of interest, the belief theory also provides source fusion support helping to extract pertinent decision from multiple sources. Generally, when sources disagree, the source fusion leads to a mass accorded to the emptyset class denoted conflict. It also represents the contradiction and conflict rate between fused sources. Thus, the conflict management has remained a compelling challenge whenever the belief function formalism was of use. ${ }^{3}$ The conflict management has been widely addressed in the literature since the introduction of Dempster's combination 
rule. ${ }^{1}$ Many works have tackled this conflict management issue proposing different types of solutions in which we can differentiate two main family approaches: (i) Conflict management approaches based on discounting the unreliable sources; $;, 5,6,7$

(ii) Redistribution of the conflict after source's combination. ${ }^{3,8}$

Discounting approaches manage the conflict by reducing the impact of a contradictory source in aggregation stage following its reliability. Even so, discounting does not eliminate contradiction which results in some mass accorded to the emptyset. Moreover, estimating source's reliability remains a difficult task.

In addition, several works on conflict redistribution families were introduced based on different heuristics. ${ }^{9,10,11,12}$ Despite their interesting conflict management results, none of the cited work stand out for its adequacy regardless the treated problem.

In this work, we aim to introduce a new conflict management called Associative Conflict Management (ACM) approach which is able to correctly handle the registered conflict by "smartly" redistributing it. In fact, several conflict management propositions were introduced. However, no approach outstanded the other ones by its versatility and adequacy independently of the addressed problem. The proposed ACM approach relies on using extra-information that would be of help for conflict management. Those information will be exploited with a data mining algorithm in order to extract only one pertinent assertion. The redistribution guided by association rules will ensure an adequate conflict management.

The paper is organized as follows. Section 2 describes the mathematical background of belief function theory for information fusion. It defines various ways to combine opinions and drawbacks of these combinations. We present the pioneering works in conflict management field and their limits are highlighted. Section 3 briefly recalls the association rule extraction fundamentals and main data mining concepts. We present the generic basis and their contribution within associative classification domain. The Associative Conflict Management (ACM) approach is thoroughly described in Section 4. We highlight the benefit of association rules introduction for conflict management through an illustrative example. Section 5 reports the encouraging results of the experimental validation of associative conflict management approach by confronting it to the classical approaches of the literature. In Section 6 , we conclude and we sketch issues of future work.

\section{Belief function theory}

The belief function theory was initiated by the work of Dempster ${ }^{1}$ on the upper and lower Probabilities. The development of the theory formalism is owed to Shafer. ${ }^{2}$ In 1976, Shafer has shown the benefits of the belief function theory to model uncertain knowledge. In addition, it allows knowledge combination obtained through various sources and offers more flexibility than do the probabilistic framework. In the remainder, we build our contribution following the Transferable Belief Model (TBM), that was introduced by Smets. ${ }^{13}$ The TBM model is an interpretation of Dempster- 
Shafer model, that aims at representing quantified beliefs based on two levels: (i) a credal level where beliefs are entertained and quantified by belief functions; (ii) a pignistic level where beliefs can be used to make decisions and are quantified by probability functions.

We present in the following, the main concepts of this theory. For further details, the interested reader is invited to refer to the work of Shafer ${ }^{2}$ or Smets. ${ }^{14}$

\subsection{Belief function Theory background}

In the following, we start by recalling the belief function theory basics.

\subsubsection{Frame of discernment}

Let us consider $\Theta$ the set of all $N$ possible exhaustive and exclusive answers for a question. $\Theta$ is called the frame of discernment and is denoted as follows:

$$
\Theta=\left\{H_{1}, H_{2}, \ldots, H_{N}\right\}
$$

From the frame of discernment $\Theta$, a super set can be deduced containing all the $2^{N}$ subsets $\mathrm{A}$ of $\Theta$ :

$$
2^{\Theta}=\{A, A \subseteq \Theta\}=\left\{\left\{H_{1}\right\},\left\{H_{2}\right\}, \ldots,\left\{H_{N}\right\},\left\{H_{1} \cup H_{2}\right\}, \ldots, \Theta\right\}
$$

This set constitutes a reference to assess the veracity of any proposal.

\subsubsection{Basic Belief Assignment (BBA)}

The Basic Belief Assignment (BBA) or the basic belief function $m$ is a function defined on each subspace of the set of disjunctions of $2^{\Theta}$ and taking values in $[0,1]$. It does not only represent all the confidence accorded to each possible response for the treated question but also the ignorance and the lack of certitude. It is represented as follows:

$$
m: 2^{\Theta} \longrightarrow[0,1]
$$

such that:

$$
\left\{\begin{array}{l}
\sum_{A \subseteq \Theta} m(A)=1 \\
m(\emptyset) \geq 0
\end{array}\right.
$$

Each hypothesis $A$ having a belief value greater than 0 is called a focal element. $m(\emptyset)$ is called the conflictual mass. A BBA is called normal whenever the emptyset is not a focal element and this corresponds to a closed world assumption, ${ }^{15}$ otherwise it is said subnormal and corresponds to an open world assumption. ${ }^{14}$ 


\subsubsection{Decision: Pignistic probability}

Decision functions allow the determination of the most suitable hypothesis from a BBA for the treated problem. In the TBM model, the pignistic level (i.e., decision level) allows decision making from usual probabilities. The pignistic probability, ${ }^{16}$ denoted $B e t P$, was proposed by Smets ${ }^{16}$ within its TBM model. Not only it does make probability transformation but it also takes into consideration the composite nature of focal elements. Formally, $\operatorname{Bet} P$ is defined as follows:

$$
\operatorname{Bet} P\left(H_{n}\right)=\sum_{A \subseteq \Theta} \frac{\left|H_{n} \cap A\right|}{|A|} \cdot \frac{m(A)}{1-m(\emptyset)} \quad \forall H_{n} \in \Theta .
$$

\subsection{Belief function theory combination operators and conflict management}

In the following, we survey several combination operators allowing source's fusion and different approaches for conflict management.

\subsubsection{Conjunctive sum}

The belief function theory combines and merges several information modelized as BBA. Several operators were defined such as the conjunctive rule. This combination operator assigns the mass to propositions initially confirmed by the majority of sources. For two sources $S_{1}$ and $S_{2}$ having respectively $m_{1}$ and $m_{2}$ as BBAs, the conjunctive sum $m_{\circledast}$ takes the following form:

$$
m_{\circledast}=m_{1} @ m_{2} .
$$

For an event $\mathrm{A}, m_{\circledast}$ can be written as follows:

$$
\left\{\begin{array}{l}
m_{\oplus}(A)=\sum_{B \cap C=A} m_{1}(B) \cdot m_{2}(C) \quad \forall A \subseteq \Theta \\
m_{\oplus}(\emptyset) \geq 0 .
\end{array}\right.
$$

where $m(\emptyset)>0$ corresponds to an open world assumption. ${ }^{14}$

\subsubsection{Orthogonal sum}

The orthogonal sum proposed by Dempster ${ }^{1}$ integrates a conflict management approach that redistributes the generated conflictual mass. It is the normalized form of the conjunctive sum rule and is defined as follows:

$$
m_{\oplus}=m_{1} \oplus m_{2} .
$$

For two sources $S_{1}$ and $S_{2}$, the aggregation of evidence can be written as follows:

$$
\left\{\begin{array}{l}
m_{\oplus}(A)=\frac{1}{1-K} \sum_{B \cap C=A} m_{1}(B) \cdot m_{2}(C)=\frac{1}{1-K} m_{\oplus}(A) \quad \forall A \subseteq \Theta, A \neq \emptyset \\
m_{\oplus}(\emptyset)=0
\end{array}\right.
$$

where $K$ is defined as:

$$
K=\sum_{B \cap C=\emptyset} m_{1}(B) \cdot m_{2}(C)=m_{\curvearrowleft}(\emptyset) .
$$




\subsubsection{Related work on conflict management}

The conjunctive sum allows the fusion of several BBAs in order to help out during the decision stage. It also generates a conflictual mass $(m(\emptyset))$ that indicates the contradiction level between fused sources but nevertheless should be eliminated. Many works have been carried out trying to propose a suitable conflict management approach. Several approaches were proposed as solutions in order to normalize BBA before and after combination process. Nevertheless, none of those solutions has distinguished by its adequacy. Three combination operator families can be differentiated proposing different methods in handling conflict. The first is mainly constituted by the conjunctive sum (see Subsection 2.2.1). This type of operator provides the best results in extracting the most truthful proposition from a multisource fusion context. However, as it is demonstrated in TBM, ${ }^{17}$ this combination has a tendency to affect resulting contradiction to the empty set.

The second family is constituted essentially of the disjunctive rule. Introduced by Dubois and Prade, ${ }^{18}$ the disjunctive combination rule generates no conflict since it redistributes the belief to composite hypothesis. Denoted $m_{\odot}$, the disjunctive rule is recommended in case of registering at least one reliable source without knowing which one. Formally, it is written as follows:

$$
m_{\odot}(A)=\sum_{B \cup C=A} m_{1}(B) \cdot m_{2}(C) \quad A \subseteq \Theta .
$$

The third family gathers all combination operators having tried to redistribute the conflict. Several works are worth of cite, such as the Yager's rule ${ }^{9}$ which redirects the entire registered conflict to the ignorance set. On the other hand, we can enumerate Dubois and Prade's DP2 ${ }^{11}$ operator that can be written as follows:

$$
m(A)=m_{\circledast}(A)+\sum_{B \cup C=A, B \cap C=\emptyset} m_{1}(B) \cdot m_{2}(C) \quad A \subseteq \Theta .
$$

Florea et al. proposed the Robust Combination Rule ${ }^{19}(\mathrm{RCR})$ that gathered in the same formula the conjunctive and the disjunctive sum, in order to profit from their respective contributions, i.e.,

$$
\left\{\begin{array}{l}
m_{R C R}(A)=\alpha(K) \cdot m_{\oplus}(A)+\beta(K) \cdot m_{@}(A) \\
m_{R C R}(\emptyset)=0
\end{array}\right.
$$

where $\alpha$ and $\beta$ are functions of the conflict $K=m_{\oplus}(\emptyset)$.

The degree of the conflict after fusion depends on many factors such that contradiction and ambiguity. ${ }^{6}$ Also, the conflict appears as an alarm indicating the contradiction between sources ${ }^{13}$ but nevertheless the decision becomes difficult. ${ }^{20}$ Indeed, in case of a high contradiction problem, making decision can be carried out only by introducing an extra-information. This extra-information could be applied in three different strategies:

- Discounting approach: the extra-information is used for estimating the reliability of the considered sources. Indeed, unreliable source are not fully 
taken into consideration during the combination stage. ${ }^{21}$

- Fusion approach: this information is considered as information source and is modeled within a belief function. All gathered sources are then combined. ${ }^{22}$

- Conflict redistribution approach: the extra-information is used to redistributes the resulting conflict to the most credible hypothesis. ${ }^{10}$

Example 1. Let us consider the problem of high-resolution classification. The image represents a forest constituted of tree crowns (see Figure 1). We aim to distinguish each tree crown class from a set of four potential tree types. Let us consider $\Theta$ the frame of discernment constituted by four classes \{Zen Oak, Cork Oak, Arboretum, Coniferous tree $\}$. The nature of the studied image has led us to consider ten different sources. Those sources can be assembled into three different groups as follows:

- Spectral information: studies the tree crown relatively to the source level of grey mean.

- Texture information: contains several information sources that analyze the tree crown by its gray level organization. The studied textural sources are Mean, Variance, Energy, Contrast and the Entropy.

- Structural information: analyzes the tree crowns following their shapes. The studied structural sources are Area, Diameter, Perimeter and the Wellepsy.

Those three groups constitute the information sources and are used in our fusion problem. In addition to those three groups, we consider another valuable but different piece of information that is:

- Spatial information: it studies the spatial disposition of the trees. The texture of the entire region is analyzed by its features.

The last information has no relation with the three others and can not be fused with them directly. In addition, it contains no further indication about the three other source's reliability. However, this information remains important and might be in help in the classification problem. Indeed, the spatial information could help out in the decision stage after fusing the three tree crown sources by redistributing the resulting conflict to the most pertinent hypothesis. In the context of conflict redistribution, the generic framework allows this kind of conflict redistribution and is presented in the following.

\subsubsection{Conflict management: Generic framework}

Several works tackled grouping conflict operator in order to profit from their complementary contributions. ${ }^{19,10,23}$ From those operators, we distinguish the Generic 


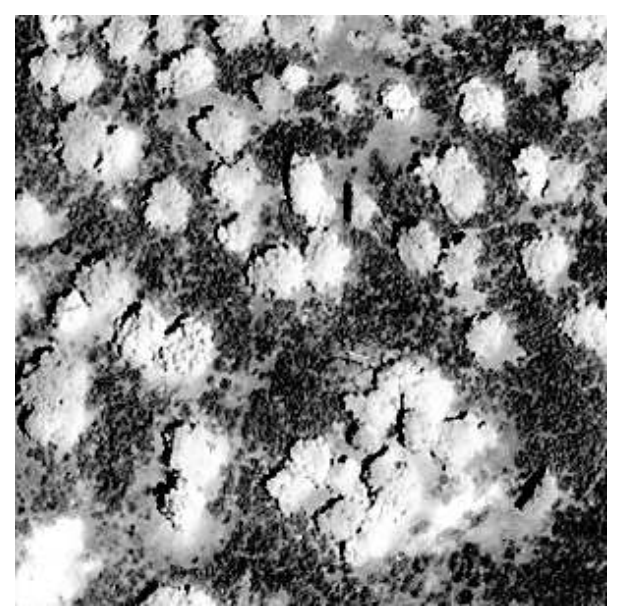

Fig. 1. Forest image sample

framework, which generalizes the conflict redistribution and unifies several redistribution rules.

Introduced by Lefevre et al., ${ }^{10}$ the generic framework aims at distributing the conflictual mass $m_{\circledast}(\emptyset)$ on a set of propositions $P$ according to a weighting factor $W(A, m)(A \subseteq P)$ with $m=\left\{m_{1}, \ldots, m_{j}, \ldots, m_{J}\right\}$. The final mass after fusion (combination), for a proposition $A$, is the sum of both masses, i.e.,

$$
\left\{\begin{array}{l}
m(A)=m_{\circledast}(A)+m^{c}(A) \quad \forall A \subseteq \Theta \\
m(\emptyset)=0 .
\end{array}\right.
$$

$m^{c}$ is part of the conflicting mass and can be written as follows:

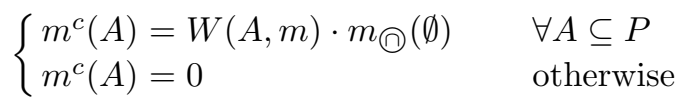

such that:

$$
\sum_{A \subseteq P} W(A, m)=1 .
$$

To resolve the problem sketched in Example 1, we propose a method for redistributing the conflict to improve the belief of the most pertinent hypothesis according to this extra-information. This operation is achievable using the generic framework which is the largest representation for any conflict management approach. In fact, it does not only provide the largest framework for conflict management but also the possibility to customize the empty set mass redistribution using the weighting factors. This characteristic makes the generic framework flexible and suited in case of existence of an additional information. 
Table 1. Extraction context $\mathcal{D}$

\begin{tabular}{lrrr}
\hline Trans ID & Attribute1 & Attribute2 & Class \\
\hline T1 & P1 & P2 & Zen Oak \\
T2 & P1 & P4 & Zen Oak \\
T3 & P1 & P2 & Zen Oak \\
T4 & P3 & P4 & Cork Oak \\
T5 & P3 & P2 & Cork Oak \\
T6 & P3 & P2 & Cork Oak \\
T7 & P1 & P2 & Zen Oak \\
T8 & P3 & P2 & Cork Oak \\
T9 & P5 & P2 & Arboretum \\
\hline
\end{tabular}

The proposed contribution relies on an additional piece of information that would help to decide where to redistribute the conflict. This information represented in a training base should be exploited in an efficient methodology to operate conflict redistribution. Since, in the literature, many methods exist allowing pertinent information's extraction from a base, we decided to use the most efficient tool performing this task. In this context, we studied data mining algorithms and associative classification which offers one of the best classification rate and measure membership. ${ }^{24}$ To introduce our approach, we present, in Section 3, data mining and associative classification principles.

\section{Data mining and associative classification}

In this section, we present the main concepts and theoretical basis within associative classification field which is based on data mining concepts.

\subsection{Mathematical Background}

Definition 1. An extraction context is a triplet $\mathcal{D}=(\mathcal{O}, \mathcal{I}, \mathcal{R})$ that represents a finite set of objects (generally called transactions) $\mathcal{O}$ and items $\mathcal{I}$ related with a binary relation (i.e., $\mathcal{R} \subseteq \mathcal{O} \times \mathcal{I}$ ). Each couple $(o, i) \in \mathcal{R}$ expresses that the object $o \in \mathcal{O}$ contains the item $i \in \mathcal{I}$. Table 1 represents a sample of an extraction context, that sketches the type of seen crowns in a region. $T_{i}$ is a transaction representing a forest region (i.e., $\mathcal{O}=\left\{T_{i} \mid i \in\{1, \ldots, 9\}\right\}$ ). The Attribute1 and Attribute2 are the features (i.e., texture characteristics) of a region $T_{i}$. Those attributes contain and indicate the existence of the set of possible items $\mathcal{I}=\{P 1, P 2, P 3, P 4, P 5\}$.

Definition 2. The support of an item $I \in \mathcal{I}$ (respectively itemset which denotes the set of several items), denoted Support(I), is defined as the proportion of transactions in the data set which contain the itemset:

$$
\operatorname{Support}(I)=\frac{|\{t \mid t \in \mathcal{O}, \forall i \in I,(t, i) \in \mathcal{R}\}|}{|\mathcal{O}|} .
$$


$I$ is called a frequent itemset if its support value is greater than or equal to a threshold minsup originally fixed by the user.

Definition 3. An association rule $R$ is defined as the implication between two itemsets. $R$ can be written as follows: $R: X \rightarrow Y$ and fulfilling those constraints $X \subset \mathcal{I}, Y \subset \mathcal{I}$ and $X \cap Y=\emptyset . X$ and $Y$ are respectively the premise and the conclusion of the rule $R$ such that $C l(R)=Y$ and $\operatorname{Pr}(R)=X$. For each rule $R$, two other measures can be defined:

- Association rule's support: it corresponds to the number of transactions $o \in$ $\mathcal{O}$ having simultaneously the premise and conclusion parts (i.e. $\operatorname{Support}(X \cup$ $Y)$ ) and is denoted Support $(R)$.

- Association rules's confidence: it expresses the conditional probability that a transaction contains $Y$ knowing that it already contains $X$ i.e. Confidence $(R: X \rightarrow Y)=\frac{\operatorname{Support}(X \cup Y)}{\operatorname{Support}(X)}$.

$R$ is an association rule of confidence if its calculated confidence is greater than or equal to a threshold minconf originally set by the user.

Example 2. Let us consider again the extraction context $\mathcal{D}$ given in Table 1. The support and the confidence of association rule $R:\{P 1, P 2\} \rightarrow$ Zen Oak are respectively equal to Support $(R)=\frac{\operatorname{Support}(\{P 1, P 2, Z \text { en Oak }\})}{|\mathcal{D}|}=\frac{3}{9}=0.33$ and Confidence $(R)=\frac{\operatorname{Support}(R)}{\operatorname{Support}(\{P 1, P 2\})}=\frac{0.33}{0.33}=1$. In other words, the computed confidence value means that if the studied region got $P 1$ and $P 2$ as features, we are sure that we are in a Zen Oak area.

Definition 4. An itemset $I \subseteq \mathcal{I}$ is said to be $\operatorname{closed}^{25,26}$ if $\omega(I)=I$ such that:

$$
\omega(I)=I \cup\{x \in \mathcal{I} \backslash I \mid S u p p o r t(I)=\operatorname{Support}(I \cup\{x\})\} .
$$

Definition 5. An itemset $g \subseteq \mathcal{I}$ is said to be a minimal generator ${ }^{27}$ of a closed itemset $f$, if and only if $\omega(g)=f$ and $\nexists g_{1} \subset g$ so that $\omega\left(g_{1}\right)=f$. The set $G_{f}$ of the minimal generators of $f$ is: $G_{f}=\left\{g \subseteq \mathcal{I} \mid \omega(g)=f \wedge \nexists g_{1} \subset g\right.$ such as $\left.\omega\left(g_{1}\right)=f\right\}$.

Example 3. Let us consider again the extraction context given in Table 1. For minsup $=0.22, P 4$ is a frequent closed item since $\omega(P 4)=P 4$ and $\operatorname{Support}(P 4)=$ $0.22 \geq$ minsup. $P 1$ and Zen $O a k$ are the minimal generators for the frequent closed itemset $\{P 1, Z$ en $O a k\}$.

The main drawback with classical association rule extraction algorithms stand in their generation of an overwhelming number of rules. ${ }^{28}$ In order to palliate this drawback, we focus our interest on the Informative Generic Base (IGB) that overcomes this fact by retaining only pertinent and generic association rules. In the following, we present the concept of generic base and $G A R C$ classifier that is build on it. 


\subsection{Informative Generic Base IGB}

Let us consider $I F F$ the set of frequent closed itemsets and $G_{c}$ the set of minimal generators of all closed frequent itemsets of $I F F$, the $I G B^{29}$ base is defined as follows:

$I G B=\left\{R: g_{s} \rightarrow\left(I-g_{s}\right) \mid I, I_{1} \in I F F \wedge I-g_{s} \neq \emptyset \wedge g_{s} \in G_{I_{1}} \wedge I_{1} \subseteq I \wedge\right.$ Confidence $(R) \geq \operatorname{mincon} f \wedge \nexists g_{1} \subset g_{s} \wedge$ Confidence $\left(g_{1} \rightarrow\left(I-g_{1}\right)\right) \geq$ minconf $\}$.

The aforementioned set $I G B$ designates the set of all association rules $R$ constructed from closed frequent itemsets $I, I_{1} \in I F F$. The premise of the rule is constituted by $g_{s} \subset I$ that is a minimal generator of $I_{1}$. Doing so, $I G B$ only retains association rules of confidence with minimal premise (i.e., rules with a larger premise are automatically dropped). Rules with empty set conclusion are also dropped. On the other hand, IGB retains the rules that maximize the conclusion part. An axiomatic system was proposed ${ }^{29}$ in order to extract the set of all valid association rules from an extraction context $\mathcal{D}$. This system fulfills two main conditions: Informativity and Derivability. ${ }^{29}$

Example 4. For minconf $=0.5$, Table 2 sketches the $I G B$ base extracted from the extraction context given by Table 1 .

Table 2. The $I G B$ rules extracted from the data set given by Table 1

\begin{tabular}{cc}
\hline Association rule & Confidence \\
\hline$\emptyset \rightarrow P 2$ & 0.78 \\
$P 1 \rightarrow\{P 2$, Zen Oak $\}$ & 0.75 \\
$Z$ en Oak $\rightarrow\{P 1, P 2\}$ & 0.75 \\
$\{P 2, P 1\} \rightarrow$ Zen Oak & 1.00 \\
$\{P 2$, Zen Oak $\} \rightarrow P 1$ & 1.00 \\
$P 1 \rightarrow$ Zen Oak & 1.00 \\
$P 3 \rightarrow\{P 2$, Cork Oak $\}$ & 0.75 \\
Cork Oak $\rightarrow\{$ P $2, P 3\}$ & 0.75 \\
$P 3 \rightarrow$ Cork Oak & 1.00 \\
$\{P 2, P 3\} \rightarrow$ Cork Oak & 1.00 \\
$\{P 2$, Cork Oak $\} \rightarrow P 3$ & 1.00 \\
\hline
\end{tabular}

In Table 2, we note the cohabitation of two types of rules: factual and implicative generic association rules. ${ }^{29}$ Indeed, a factual generic association rule, fulfilling the premise part emptiness, allows one to highlight item correlations without any condition. However, for an implicative generic association rule, where the premise part is not empty, item correlations are conditioned by the existence of those of the premise items. 


\subsection{Associative classification generic rules}

Several associative classification approaches were proposed. ${ }^{30,31} G A R C$ is an associative classifier, initially proposed by Bouzouita et al., that extracts the generic classification rules directly from a generic base of association rules. ${ }^{24}$ This characteristic allows to avoid the generation of a large number of associative classification rules (main drawback of other approaches). GARC filtrates generic rules to retain only whose conclusion's part includes a class label. Thanks to the $I G B$ base, the classification rules extracted by $G A R C$ impose less constraints which is owed to the fact the premise part is as small as possible.

Example 5. Table 3 sketches the classification rules extracted from the $I G B$ base given in Table 2 .

Table 3. The Classification rules extracted from $I G B$ base

\begin{tabular}{cc}
\hline Association rule & Confidence \\
\hline$P 1 \rightarrow$ Zen Oak & 1 \\
$\{P 1, P 2\} \rightarrow$ Zen Oak & 1 \\
$P 3 \rightarrow$ Cork Oak & 1 \\
$\{P 3, P 2\} \rightarrow$ Cork Oak & 1 \\
\hline
\end{tabular}

\section{Associative Conflict Management approach: ACM}

In the following, we introduce the Associative Conflict Management (ACM) approach allowing a smart conflict redistribution. The proposed ACM approach relies on the generic base (defined above) for subnormal BBAs treatment.

\subsection{Approach Presentation}

The basic idea of our conflict management approach is based on allowing an automatic and adequate conflict redistribution. We constructed our contribution using the generic framework (c.f Subsection 2.2.4), which formalizes any redistributing conflict management approach proposed in the literature. Finding the correct weighting factors for any BBA will ensure a better redistribution. In this respect, their determination are provided using the classification association rules.

The first part of our approach consists in finding the Generic association rules. As depicted in Figure 2, from a training base, we construct our extraction context. The application of $G A R C$ algorithm provides a set of classification rules fitted with a confidence measure. Every association rule constitutes a valuable piece of information in defining the amount of conflict that should be assigned to a specific class. The main steps of our approach is that the confidence of an association rule is the extra-information that would be of help for defining rates of conflict distribution. Indeed, each rule indicates the pertinence of redistributing conflict to 


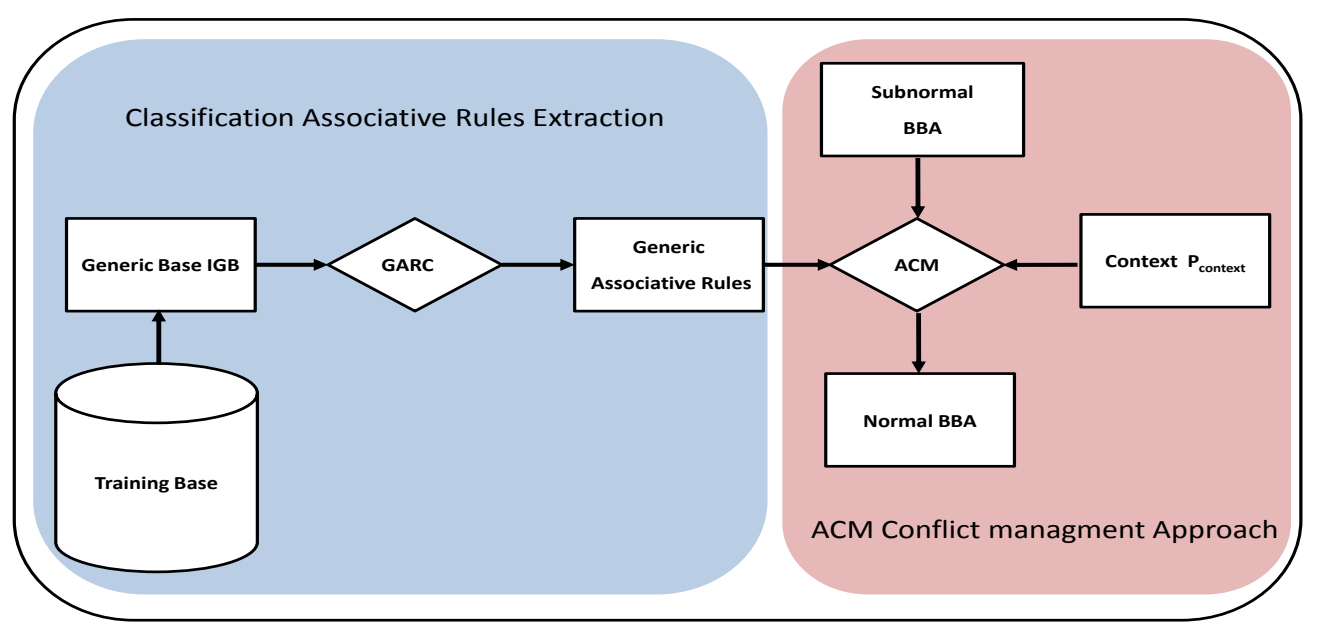

Fig. 2. The proposed architecture for associative conflict manager

a class (constituting the rule conclusion) according to the studied context (defined by the rule's premise). For that reason, in Figure 2, ACM takes as inputs a subnormal BBA resulting from source's fusion, the set of classification rules and features relative to the studied scene. Those features are used to select the rule to use for conflict redistribution on the subnormal BBA. In order to avoid a high number of association rules given by the common rule extraction algorithms, we opted to work with a generic base. The generic base's contribution is undeniable, since the latter proposes only pertinent and a restrained number of association rules.

\subsection{Associative Weighting factors}

In the following, we present our method to determine the weighting factor of the generic framework.

Definition 6. Let us consider a subnormal belief function $m$ evolving in the frame of discernment $\Theta$ and an extraction context $\mathcal{D}$. Additionally, we consider $P_{\text {context }}$, the extra-information regarding the same treated scene needed for conflict management. $P_{\text {context }}$ can be considered as a fixed size itemset such that $\forall i, i \subseteq P_{\text {context }}, i \subseteq \mathcal{I}$. Thanks to $P_{\text {context }}$, which represents the context measure for a source $m$, we can manage the conflict as follows:

$\left\{\begin{array}{l}W_{\text {ass }}(C, m)=\max \left\{\text { Confidence }(R)\left|\nexists R^{\prime}\right| \frac{\operatorname{Pr}\left(R^{\prime}\right)}{P_{\text {context }}}|>| \frac{\operatorname{Pr}(R)}{P_{\text {context }}} \mid \wedge C l(R)=C \wedge C l\left(R^{\prime}\right)=C\right\} \\ W_{\text {ass }}(\Theta, m)=1-\text { Confidence }(R) .\end{array}\right.$

We can remark from the previous formula that the redistribution will involve only 
two focal elements. The first class is the one which exists in the conclusion part of a valid association rule maximizing the premise constraint. The confidence of this rule corresponds then to the weighting factor of the conclusion class. If the found rule is not exact (Confidence $<1$ ), then the rest of conflict will be assigned to ignorance class $\Theta$. In the case where no matching rule is found, all conflict will be redistributed to the ignorance $\Theta$. Thus, the ACM is assimilated to Yager's rule. ${ }^{9}$ We can clearly see from the following formula that the unicity constraint is fulfilled:

$$
\sum_{A \subseteq \Theta} W_{a s s}(A, m)=1 \text {. }
$$

The generic framework can be written as follows:

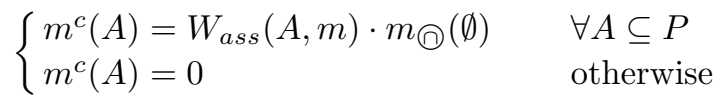

where $P$ defines the set containing the class of the found association rule (i.e. $C l(R))$.

Example 6. In this example, we study again the effect of our approach on the tree crown classification example (see Example 1). To treat the registered conflict with our ACM approach, we are equipped with an extraction context $\mathcal{D}$ containing some tree crown spatial information records (Table 1). Let us consider the following conflictual BBA $m$ evolving in the frame of discernment $\Theta=$ $\{$ Zen Oak, Cork Oak, Arboretum $\}$ :

$$
\begin{cases}m_{\circledast}(\{\text { Arboretum }\}) & =0.0001 \\ m_{\circledast}(\emptyset) & =0.9999 .\end{cases}
$$

In the training base, we have the $P 1$ and $P 2$ features (i.e., $P_{\text {context }}=\{P 1, P 2\}$ ). From the generated rules, we notice the following one $R 1: P 1 \rightarrow$ Zen Oak which is more generic than the rule $R 2:\{P 1, P 2\} \rightarrow Z$ en Oak and shares the same confidence value $100 \%$. The latter means in terms of weighting factors:

$$
W_{\text {ass }}(\{\text { Zen } O a k\}, m)=1
$$

Considering this constraint, the BBA $m$ becomes after the ACM treatment:

$$
\begin{cases}m(\{\text { Zen Oak }\}) & =W_{\text {ass }}(\{\text { Zen Oak }\}, m) \cdot m_{\circledast}(\emptyset) \\ m(\{\text { Arboretum }\}) & =m_{\circledast}(\{\text { Arboretum }\})+W_{\text {ass }}(\{\text { Arboretum }\}, m) \cdot m_{\circledast}(\emptyset) \\ m(\emptyset) & =0\end{cases}
$$

that finally gives:

$$
\begin{cases}m(\{\text { Zen Oak }\}) & =0.9999 \\ m(\{\text { Arboretum }\}) & =0.0001 \\ m(\emptyset) & =0 .\end{cases}
$$

\section{Experiments and results}

In order to validate the proposed approach, we conducted experiments on highresolution remote sensing image, putting the focus on the problem of tree crown classification. 


\subsection{Distance estimation model classifier}

In order to profit from the large amount of data constituting our learning base, we opted for the Zouhal et al. ${ }^{32}$ distance BBA estimation model based on a distance measure. The presence of a training pattern $x^{i}$, having the class $\left\{H_{n}^{i}\right\}$, among the $k$ Nearest Neighbors (KNN) of under classification pattern $x$ is considered as a piece of evidence. Indeed, it influences our belief concerning the class membership of the entity under consideration. This information is represented by a BBA $m$ over the set $\Theta$ of classes. A fraction of the unit mass is assigned by $m$ to the singleton $\left\{H_{n}^{i}\right\}$, and the remaining is assigned to the whole frame of discernment $\Theta$. The mass $m\left(\left\{H_{n}^{i}\right\}\right)$ is defined as a decreasing function of the distance $d$ between $x$ and $x^{i}$ in the feature space. The $k$ nearest neighbors of $x$ can be regarded as $k$ independent sources of information represented by BBA. These several pieces of evidence can be aggregated by means of Dempster's combination rule to represent our belief concerning $x$ class membership.

The adopted strategy consists in modeling the information according to every characteristic $x_{j}$ (with $j \in[1, J]$ ) of the vector $x$ to classify. A belief function $m_{s j}$ is then defined by: ${ }^{33}$

$$
\left\{\begin{array}{l}
m_{s j}\left(\left\{H_{n}\right\}\right)=\alpha_{j}^{s} \phi_{j}^{s}\left(d_{j}^{s}\right) \\
m_{s j}(\Theta)=1-\alpha_{j}^{s} \phi_{j}^{s}\left(d_{j}^{s}\right)
\end{array}\right.
$$

where $0<\alpha_{j}^{s}<1$ is a constant, $d_{j}^{s}$ represents the distance between the $j$-th component $x_{j}$ of the vector $x$ and its neighboring vector $v_{s}(s \in[1, k])$. The function $\phi_{j}^{s}$ can be expressed in the following way:

$$
\phi^{s}\left(d_{j}^{s}\right)=\exp \left(-\gamma_{j}^{s}\left(d_{j}^{s}\right)^{2}\right) .
$$

A learning algorithm was proposed by Zouhal et al. ${ }^{32}$ for computing the parameters $\gamma_{j}^{s}$ in the Eq. (19) by optimizing an error criterion.

Dempster's combination is used to combine those $k$ belief functions. $m_{j}$ is the resulting belief function and it is equal to:

$$
m_{j}=\oplus_{s \in[1, k]} m_{s j}
$$

Thanks to its two hypothesis constructed BBA (see Eq. (18)), this model avoids combinatorial explosion resulting from several fusion processes. A unique belief function $m$ is obtained by the application of the same fusion principle on those resulting $J$ BBAs:

$$
m=\oplus_{j \in[1, J]} m_{j}
$$

with $J$ standing for number of sources.

\subsection{ACM classifier for tree crowns classification}

In the following, we introduce the ACM classifier for tree crown identification. We integrated the Associative Conflict Management in the distance estimation model 
Table 4. Registered conflict for studied tree crowns

\begin{tabular}{rrrr}
\hline Conflict rate & {$[\mathbf{0 , 0 . 2 )}$} & {$[\mathbf{0 . 2}, \mathbf{0 . 4})$} & {$[\mathbf{0 . 4}, \mathbf{1})$} \\
\hline & $12 \%$ & $14 \%$ & $74 \%$ \\
\hline
\end{tabular}

initially presented in Section 5.1. We highlight, in the following, the adaptation of the distance model in a tree crown classification context.

Let us consider $\Theta$ the frame of discernment constituted by four classes $\{$ Zen Oak,Cork Oak, Arboretum, Coniferous tree $\}$. As it is already mentioned in Example 1, four different information sources are distinguished. The Spectral, Texture and the Structural sources are used in the source fusing problem while the Spatial one is for the conflict redistribution.

For each one of the three information fusing source, we apply a $K \mathrm{NN}$ belief function estimation (Eq. (18)). Each feature, gives four BBAs $(k=4)$ which are combined via the Dempster's combination rule (Eq. (20)). The result is a single BBA expressing the crown membership from the point of view of the considered source. The gathered ten source's BBA are also combined through (Eq. (21)) to get the final tree crown's BBA. In the sequel, the described Distance Model Classifier is denoted DMC. Interested reader may refer to ${ }^{34}$ for further details.

To achieve the ACM treatment, we replaced the orthogonal sum in Eq. (21) by the conjunctive one (Eq. (5)). The modification aims to expose the conflict existing between combined sources and prepare it for an ACM treatment. The higher the conflict value is, the more effective ACM becomes. Table 4 shows the registered conflict after a conjunctive combination. Indeed, $74 \%$ of considered trees present a conflict higher than 0.4 , which means potentially class changeable with an adequate conflict manager. In our work, to handle the conflict, an extra-information is required. Unlike during the modelization stage where information were collected from tree crowns, the extra-information base is constructed from image regional analysis. Since, each tree specie obeys to a natural organization presence, studying those areas can be considered as a valuable information for decision making. For that reason, texture information are collected from the image for each one of the four considered classes. As we can notice, this kind of information has no indication about reliability neither can be fused with other sources. For those reasons, the use of ACM is appropriate. To realize this task, GARC is applied on the collected information in order to extract generic rules necessary for conflictual BBA treatment.

\subsection{Generic base and associative classifier contribution}

In following, we present experimentally an assessment of the generic base contribution. In order to evaluate the contribution of the $I G B$ base in associative classification, we are interested in comparing its performance to associative classifier CBA. ${ }^{31}$ $\mathrm{CBA}$ is a classifier which filtrates association rules resulting from the Apriori algorithm. In fact, Apriori is a two steps algorithm that generates all frequent itemset 


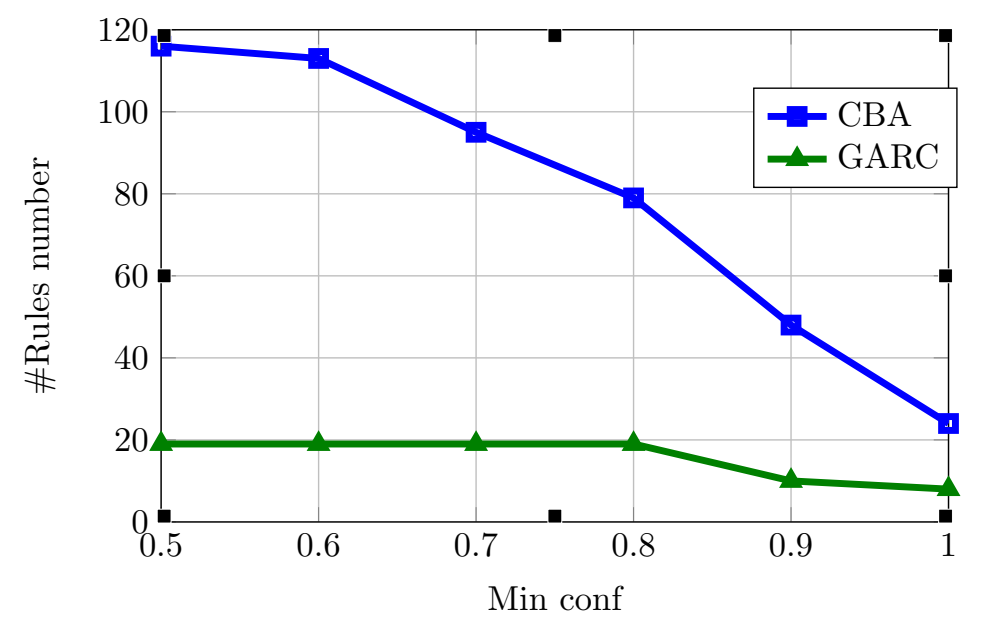

Fig. 3. Number of associative classification rules for minsup $=0.1$

satisfying the minimum support constraint. From extracted frequent itemsets, Apriori generates all possible association rules. Interested reader is invited to refer to Agrawal's work. ${ }^{28}$ To demonstrate the contribution of the generic base, CBA and $G A R C$ were compared in Figure 3 in terms of the generated rule's number. The tests were conducted on a base of 564 instances. Each instance is extracted from the image and represents a regional textural information.

The $I G B$ provides not only a fewer number of association rules (see Figure 3), but also retains association rules of high quality. Comparatively to CBA, the $I G B$ generic base has dropped redundant rule. Indeed several rules can be dropped for only one having a shorter premise and more generecity.

The GARC classifier also points out competitive result comparatively to other known works. Experiments were conducted on benchmarks proving the efficiency of $G A R C .{ }^{24}$ In fact, it provides a better results than other classification approaches such that decision tree. In our case, GARC not only provides a good performance rate but also class membership value to each classified instance. This value expressed by the confidence rate is an additional information that cannot be neglected.

\subsection{Classification and conflict management contribution}

The ACM addition in conflict management is proven by comparing the proposed approach vs. the well known approaches of the literature. The proposed ACM approach was tested on conflictual BBA resulting from distance modeling. Those BBA represent 399 tree crowns. The results were compared to a Distance Model Classifier (DMC) based on the distance belief estimation model and Dempster's combination (see Subsection 5.1). We also compared the ACM method to a Distance Model Classifier that integrates the extra-information in the combination (DMCE 
in the Table 5). A BBA can be modeled by the distance classifier (see Subsection 5.1). However, in this case, instead of using a training base, we represented classes by prototypes. ${ }^{33}$ Since the spatial information represents features of a forest area, its frame of discernment is different from the tree crown's information sources. The frame of discernment for this information, noted $\Theta_{r}$, is defined by $\Theta_{r}=\left\{Z_{\text {en Oak }}\right.$, Cork Oak $k_{r}$, Arboretum $_{r}$, Coniferous tree $\left._{r}\right\}$. Since a forest area contains several trees, a vacuous extension can be conducted on each constructed BBA in order to unify frames of discernment. This vacuous extension of a BBA $m$ from a frame of discernment $\Theta_{r}$ to $\Theta$ can be written as follows:

$$
m^{\Theta_{r} \downarrow \Theta}(\rho(B))=m^{\Theta_{r}}(B) \quad \forall B \subseteq \Theta_{r} .
$$

where $\rho$ is the transforming of $\Theta_{r}$ to $\Theta$ and defined by:

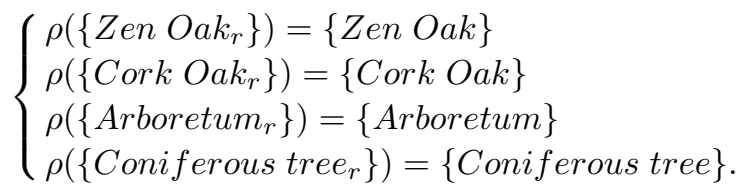

Now, the BBAs are defined on the same frame of discernment and they can be combined. This operation (vacuous extension) can not work if the studied area contains heterogeneous types of trees.

Table 5. Performance Comparative results: ACM vs DMC

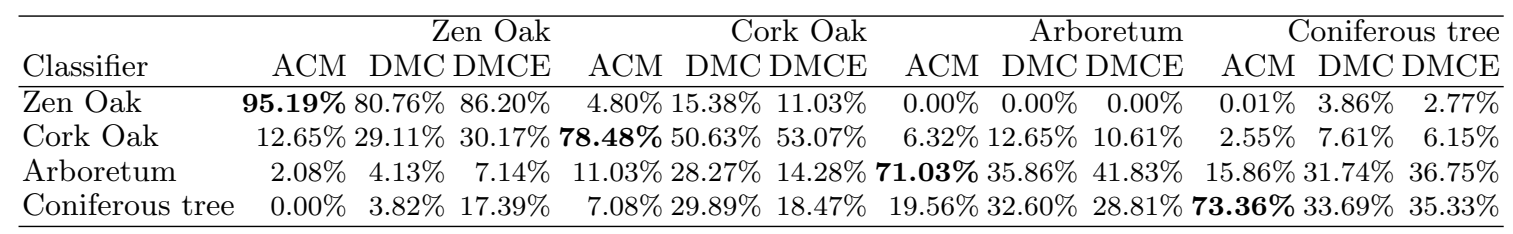

As shown in Table 5, the ACM approach presents better results than those provided by the DMC approach. Thanks to its contextual conflict management, ACM has considerably improved classification for all considered classes. The improvement shows the importance of using the spatial information within the classification process. We also had improved the result comparatively to DMCE approach which shows the benefit of using the extra-information as we did. Since we have no indication about the spatial information reliability, it is fused with the other sources with same weight. For this reason, comparatively to DMC, DMCE improvement is small.

Figure 4 illustrates an area that contains only Zen tree species classified with a DMC approach. In this image, the Zen Oak, Cork Oak, Arboretum and Coniferous tree are respectively colored in green, red, blue and yellow. Comparatively to Figure 5 , that was classified with an ACM approach, we remark that we improved the classification where several Cork Oak and Arboretum trees has changed to Zen 


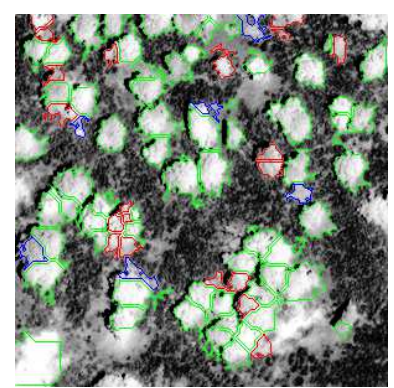

Fig. 4. Zen area DMC classification.

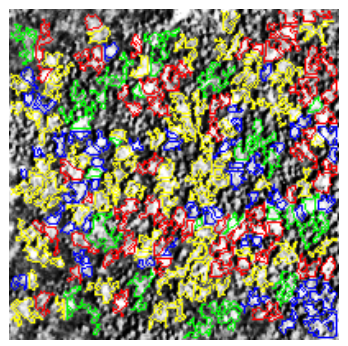

Fig. 6. Coniferous tree area DMC classification.

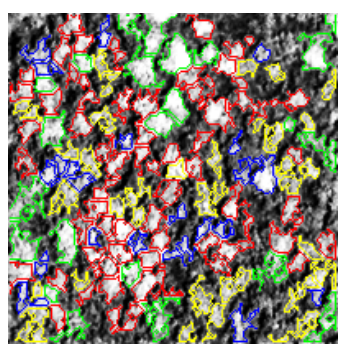

Fig. 8. Cork Oak area DMC classification.

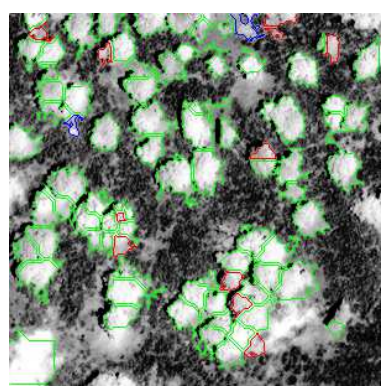

Fig. 5. Zen area ACM classification.

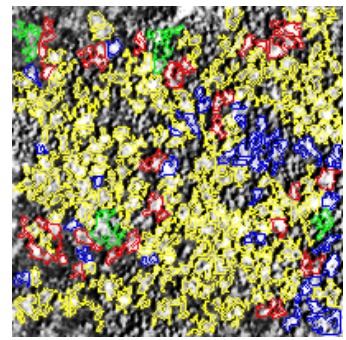

Fig. 7. Coniferous tree area ACM classification.

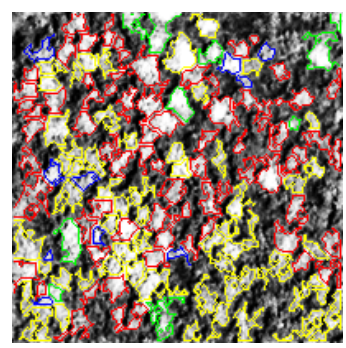

Fig. 9. Cork Oak area ACM classification. 
Oak as it is actually the case. The experiments were also conducted on typical Coniferous and Cork Oak area (see Figures 6, 7, 8 and 9) where good classification has significantly improved using ACM methods. Indeed, the use of generic rules has improved results by modifying BBA with a suited conflict management. Even, if a BBA identifies a wrong class after source fusion, the association rule can change that by redirecting conflict to the adequate class leading to classification improvement.

\section{Conclusion}

In this paper, we introduced a new method for conflict management based on conflict redistribution. We have shown that for some fusion cases, a typical conflict management is not adequate and an extra-information is needed. We propose a redistribution conflict management approach that exploits this additional information. This extra-information is integrated via the generic rules. Those generic rules are used to compute the pertinence of each conflict redistribution. For validation, we demonstrated the importance of using the generic rules and their classification impact. We also experimented our approach on a high-resolution remote sensing image. In future work, the association rule contribution can be investigated further more by studying the impact of fuzzy rules. Indeed, in our case, we proceeded to data discretization before applying any data mining approach. We could study fuzzy data mining approaches which could treat those kinds of numerical data. Additionally, the integration of association rules may be interesting in other belief function theory domain such that estimating source reliability, discounting, etc. Indeed, in several problems, the additional information represented by association rules can be used to estimate each source reliability.

\section{References}

1. A. Dempster, Upper and lower probabilities induced by multivalued mapping, AMS38, 1967.

2. G. Shafer, A Mathematical Theory of Evidence, Princeton University Press, 1976.

3. K. Sentz, S. Ferson, Combination of evidence in Dempster-Shafer theory, Tech. rep. (2002).

4. J. Klein, O. Colot, Automatic discounting rate computation using a dissent criterion, in Proceedings of the Workshop on the Theory of Belief Functions, Brest, France (2010) 1-6.

5. A. Martin, A.-L. Jousselme, C. Osswald, Conflict measure for the discounting operation on belief functions, in Proceedings of 11th International Conference on Information Fusion, Cologne, Germany (2008) 1003-1010.

6. A. Samet, E. Lefevre, S. Ben Yahia, Reliability estimation with extrinsic and intrinsic measure in belief function theory, In Proceedings of the 5th International Conference on Modeling, Simulation and Applied Optimization (ICMSAO13), April 28-30 Hammamet, Tunisia (2013) 1-6.

7. J. Schubert, Conflict management in Dempster-Shafer theory using the degree of falsity, International Journal of Approximate Reasoning 52 (3) (2011) 449-460.

8. P. Smets, Analyzing the combination of conflicting belief functions, Information Fusion 8 (4) (2007) 387-412. 
9. R. Yager, On the Dempster-Shafer framework and new combination rule, Information Sciences 41 (1987) 93-138.

10. E. Lefevre, O. Colot, P. Vannoorenberghe, Belief functions combination and conflict management, Information Fusion 3 (2002) 149-162.

11. D. Dubois, H. Prade, Representation and combination of uncertainty with belief functions and possibility measures, Computational Intelligence 4 (1988) 244-264.

12. F. Smarandache, J. Dezert, Proportional conflict redistribution rules for information fusion, advances and applications of DSmT for information fusion (collected works), American Research Press, Rehoboth Vol. 2 (2006) 3-68.

13. P. Smets, The Transferable Belief Model and other Interpretations of DempsterShafer's Model, In Proceedings of 6th Annual Conference on Uncertainty in Artificial Intelligence, UAI, Amsterdam, NL (1990) 375-383.

14. P. Smets, R. Kennes, The Transferable Belief Model, Artificial Intelligence 66 (2) (1994) 191-234.

15. P. Smets, Belief functions, in Non Standard Logics for Automated Reasoning, P. Smets, A. Mamdani, D. Dubois, and H. Prade, Eds. London,U.K: Academic (1988) 253-286.

16. P. Smets, Decision making in the TBM : The necessity of the pignistic transformation, International Journal of Approximate Reasoning 38 (2005) 133-147.

17. P. Smets, The combination of evidence in the Transferable Belief Model, IEEE Transactions on Pattern Analysis and Machine Intelligence 12 (5) (1990) 447-458.

18. D. Dubois, H. Prade, On the unicity of Dempster rule of combination, Int. J. Intelligent Systems 1 (1986) 133-142.

19. M. Florea, A.-L. Jousselme, E. Bossé, D. Grenier, Robust combination rules for evidence theory, Information Fusion 10 (2009) 183 - 197.

20. E. Lefevre, Z. Elouedi, How to preserve the conflict as an alarm in the combination of belief functions?, Decision Support Systems 56 (2013) 326-333.

21. D. Mercier, T. Denoeux, M. H. Masson, Belief function correction mechanisms, in: B. Bouchon-Meunier, al (Eds.), Foundations of Reasoning under uncertainty, Vol. 249 of Studies in Fuzziness and Soft Computing, Springer Berlin Heidelberg, 2010, pp. 203-222.

22. Z. Elouedi, E. Lefevre, D. Mercier, Discounting of a belief function using a confusion matrix, in Proceedings of the 22th IEEE International Conference on Tools with Artificial Intelligence, ICTAI'2010, Arras, France (2010) 287-294.

23. E. Lefevre, Z. Elouedi, D. Mercier, Towards an alarm for opposition conflict in a conjunctive, European Conference Symbolic and Quantitative Approaches to Reasoning with Uncertainty, ECSQARU'2011, Springer, LNCS 6717, Belfast, UK (2011) 314325 .

24. I. Bouzouita, S. Elloumi, S. Ben Yahia, GARC: A new associative classification approach, in Proceedings of 8th International Conference Data Warehousing and Knowledge Discovery, DaWaK, Krakow, Poland (2006) 554-565.

25. N. Pasquier, Y. Bastide, R. Taouil, L. Lakhal, Efficient mining of association rules using closed itemset lattices, Journal of Information Systems 24 (1999) 25-46.

26. L. Lakhal, G. Stumme, Efficient mining of association rules based on formal concept analysis, in: G. Bernhard, S. Gerd, W. Rudolf (Eds.), Formal Concept Analysis, Vol. 3626 of Lectures Notes in computer Science, Springer Berlin Heidelberg, 2005, pp. 180-195.

27. Y. Bastide, N. Pasquier, R. Taouil, L. Lakhal, G. Stumme, Mining minimal nonredundant association rules using frequent closed itemsets, In Proceedings of the International Conference DOOD2000, LNAI Springer-Verlag, London, UK volume 1861 
(2000) 972-986.

28. R. Agrawal, R. Srikant, Fast algorithm for mining association rules, In Proceedings of international conference on Very Large DataBases, VLDB, Santiago de Chile, Chile (1994) 487-499.

29. S. B. Yahia, G. Gasmi, E. M. Nguifo, A new generic basis of "factual" and "implicative" association rules, Intell. Data Anal. 13 (4) (2009) 633-656.

30. W. Li, J. Han, J. Pei, CMAR: Accurate and efficient classification based on multiple class-association rules, in Proceedings of IEEE International Conference on Data Mining (ICDM01), San Jose, CA, IEEE Computer Society (2001) 369-376.

31. B. Liu, W. Hsu, Y. Ma, Integrating classification and association rule mining, in Proceedings of the 3th International Conference on Knowledge Discovery and Data Mining, KDD, New York City, New York (1998) 80-86.

32. L. Zouhal, T. Denoeux, An evidence-theoretic K-NN rule with parameter optimization, IEEE Transactions on Systems, Man and Cybernetics, Part C 28 (2) (1998) $263-271$

33. T. Denoeux, A neural network classifier based on Dempster-Shafer theory, IEEE Transactions on Systems, Man and Cybernetics, Part A 30 (2) (2000) 131-150.

34. A. Samet, Z. B. Dhiaf, A. Hamouda, E. Lefevre, Classification of high-resolution remote sensing image by adapting the distance belief function estimation model, in Proceedings of International Conference on Communications, Computing and Control Applications, CCCA'2011, Hammamet, Tunisia (2011) 1-6. 